\title{
Modelado estadístico para la gestión de los primeros tres meses de la COVID-19 en Costa Rica ${ }^{1}$
}

\author{
Statistical Modeling for the Management of the Initial State \\ of Emergency: First Three Months of COVID-19 in Costa Rica
}

\author{
Modelagem estatística para a gestão \\ inicial de emergências: os primeiros três meses \\ de COVID-19 na Costa Rica
}

\section{Guaner Rojas}

Universidad de Costa Rica. San José, Costa Rica guaner.rojas@ucr.ac.cr

https://orcid.org/0000-0002-3064-9631

Ronny Pacheco

Universidad de Costa Rica. San José, Costa Rica ronny.pacheco@ucr.ac.cr https://orcid.org/0000-0002-1448-1058

\section{Rodolfo Romero}

Universidad de Costa Rica. San José, Costa Rica rodolfo.romero@ucr.ac.cr https://orcid.org/0000-0002-8601-5750

\section{Carlos Villalobos}

Universidad de Costa Rica. San José, Costa Rica

carlos.villalobosaraya@ucr.ac.cr

https://orcid.org/0000-0001-5003-6130

\section{Agustín Gómez \\ Universidad de Costa Rica. San José, Costa Rica agustin.gomez@ucr.ac.cr \\ https://orcid.org/0000-0002-7383-3754}

DOI: https://doi.org/10.32719/25506641.2021.10.3

Recibido: 26 de febrero de $2021 \cdot$ Revisado: 1 de marzo de 2021

Aceptado: 10 de marzo de $2021 \cdot$ Publicado: 1 de julio de 2021

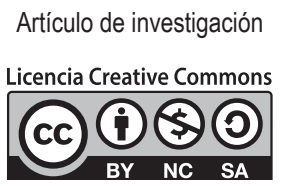

1. Este artículo se ha desarrollado en el marco de una investigación activa formalmente inscrita en la Universidad de Costa Rica en el que participan siete unidades académicas y 12 investigadores, 


\section{Resumen}

El papel del modelado estadístico en la gestión de emergencias es fundamental para perfilar o apoyar las decisiones en torno a la atención de los eventos. En 2020 con el surgimiento de la pandemia por coronavirus, los países rápidamente se prepararon para la atención del comportamiento de contagio y el impacto que tendría en la salud pública. En Costa Rica, un equipo de especialistas preparó estudios sobre el comportamiento de la curva de contagio y su efecto en la ocupación de camas hospitalarias durante los primeros tres meses de la presencia de la epidemia. Los estudios se basaron en la estimación de modelos estadísticos de crecimiento exponencial y logístico, los cuales proporcionaron los pronósticos del número de casos diarios y acumulados. La predicción de casos permitió alimentar un modelo de simulación para la proyección de demanda de camas hospitalarias por pacientes de la COVID-19. Los análisis se basaron en los datos aportados por el Ministerio de Salud en torno a los casos confirmados por coronavirus desde la aparición del primer caso en Costa Rica. Se estimaron cuatro modelos: logístico, Richards, Gompertz y exponencial, los cuales generaron la predicción de casos diarios. También se estimó el número de reproducibilidad mediante estadística bayesiana para cuantificar la transmisibilidad del virus. Los resultados permitieron anticipar el comportamiento inicial del virus en Costa Rica y el potencial efecto de las medidas de contención que se adoptaron a partir de la declaratoria de emergencia nacional.

Palabras clave: coronavirus, COVID-19, logístico, exponencial, reproducibilidad.

JEL: C15 Métodos de simulación estadística.

\section{Abstract}

The role of statistical modeling in emergency management is essential to shape or support decisions regarding the care of events. In 2020 with coronavirus pandemic, countries quickly braced themselves for attention to contagious behavior and the impact it would have on public health. In Costa Rica, a team of specialists prepared studies on the behavior of the contagion curve and its effect on the occupation of hospital beds during the first three months of the epidemic. The studies were based on statistical model estimation of exponential and logistic growth, which provided forecasts of the daily and accumulated case numbers. The prediction of cases allowed feeding a simulation model for the projection of demand for hospital beds by patients with COVID-19. The analysis were based on data provided by the Ministry of Health regarding confirmed cases of coronavirus since the appearance of the first case in Costa Rica. Four models were estimated: logistic, Richards, Gompertz and Exponential, which generated daily case predictions.

en el marco de la pandemia de la COVID-19 y en trabajo interinstitucional con el Ministerio de Salud de Costa Rica, la Organización Panamericana de la Salud y otras instituciones. Bajo esta perspectiva se justifica la autoría colaborativa de al menos cinco investigadores que participaron desde el mes de marzo de 2020 en esta línea de investigación y a la que se refiere el presente artículo. En el vínculo que está a continuación podrán encontrar el registro de la correspondiente investigación en la Universidad de Costa Rica: https://vinv.ucr.ac.cr/sigpro/web/projects/C0245. 
The reproducibility number was also estimated using Bayesian statistics to quantify the virus transmissibility. The results made it possible to anticipate the initial behavior of the virus in Costa Rica and the potential effect of the containment measures adopted after the declaration of national emergency.

Keywords: coronavirus, COVID-19, logistic, exponential, reproducibility.

JEL: C15 Statistical simulation methods: general.

\section{Resumo}

O papel da modelagem estatística na gestão de emergências é fundamental para delinear ou apoiar as decisões em torno da resposta aos eventos. Em 2020, com o surgimento da pandemia do coronavírus, os países rapidamente se prepararam para responder ao comportamento de contágio e para o impacto que provocaria sobre a saúde pública. Na Costa Rica, uma equipe de especialistas preparou estudos sobre o comportamento da curva de contágio e seu efeito sobre a ocupação de leitos hospitalares durante os primeiros três meses da epidemia. Os estudos se basearam na estimativa de modelos estatísticos de crescimento exponencial e logístico, os quais proporcionaram prognósticos quanto ao número de casos diários e acumulados. A predição de casos permitiu alimentar um modelo de simulação para a projeção da demanda de leitos hospitalares por pacientes com COVID-19. As análises se basearam em dados fornecidos pelo Ministério de Saúde em relação aos casos confirmados de coronavírus a partir do aparecimento do primeiro caso na Costa Rica. Estimaram-se quatro modelos: logístico, Richards, Gompertz e exponencial, os quais geraram a estimativa de casos diários. Estimou-se também o número de reprodutibilidade por meio da estatística bayesiana de forma a quantificar a transmissibilidade do vírus. Os resultados permitiram antecipar o comportamento inicial do vírus na Costa Rica e o potencial efeito das medidas de contenção adotadas a partir da declaração de emergência nacional.

Palavras chave: COVID-19, logístico, exponencial, reprodutibilidade, modelos. JEL: C15 Métodos de simulação estatística.

\section{Introducción}

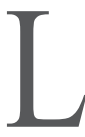

os primeros casos ocurridos por el virus SARS-CoV-2 en China iniciaron la diseminación de la enfermedad coronavirus (COVID-19) por diversos países. El virus se transmitió entre las personas con tanta rapidez que en poco tiempo varios países empezaron a contar casos y a tomar acciones para ralentizar el contagio. En Costa Rica el primer caso por coronavirus se confirmó el 6 de marzo de 2020 (Chaves et al. 2020), proveniente de un vuelo comercial desde Estados Unidos, mientras que para esa fecha en varios países europeos como Italia ya se registraban un acumulado que 
superaba los 1000 casos, e inclusive estaban contabilizando defunciones asociadas a la COVID-19.

Diversos estudios analizaron datos de distintos países y los resultados han determinado que bajo ciertas circunstancias el comportamiento del crecimiento de casos sigue formas exponenciales y logísticas (Roosa et al. 2020). En la fase inicial de la pandemia en varios países se evidenció un comportamiento esperado según una tendencia exponencial cuando no se adoptaron medidas de mitigación de la transmisión e impacto del contagio, lo cual coincide con los comportamientos exponenciales y subexponenciales esperados en la caracterización inicial de epidemias (Chowell et al. 2016).

La incidencia, o casos nuevos diarios, por el brote de coronavirus es información disponible y reportada por las entidades de salud pública desde la aparición del primer caso en Costa Rica, donde en las primeras etapas de la pandemia se proporcionó información de una manera muy básica mediante infografías y posteriormente mediante plataformas más colaborativas de socialización de información. Sin embargo, en la toma de decisiones para la previsión del impacto del virus se requiere un pronóstico de los casos esperados para días posteriores. El pronóstico toma relevancia para la planificación de acciones de contención que minimicen el impacto tal como la preparación y equipación de los hospitales con las camas y unidades de cuidados intensivos necesarios para dar respuesta al internamiento de pacientes por la COVID-19 (Torabipour et al. 2016).

El modelado estadístico para la proyección de los casos esperados se realizó bajo supuestos que se establecen en función de la complejidad y acción de los modelos. En determinadas situaciones y supuestos se consideraron factores a nivel de modelado que ayudan a seleccionar un modelo que describe en cierta medida el comportamiento actual para poder proyectar a futuros casos.

En Costa Rica, en el estado inicial del contagio la cantidad de casos confirmados fueron pocos, lo cual limitó el uso de modelos epidemiológicos o alguna adaptación que permitiera generar una proyección de casos a partir de información disponible y con supuestos en el contexto de medidas de contención. En general, la cantidad de casos reportados se mantuvo constante y con pocos casos diarios durante los primeros 100 días de la pandemia (CR Ministerio de Salud 2021). 
El lento crecimiento de casos provocado por las medidas de contención y la falta de acceso a datos, debido a la confidencialidad de estos, provocaron que los primeros análisis se basaran en modelos que capturaran el comportamiento de los casos acumulados por la COVID-19 y no en el comportamiento diario. También, si se asume que las medidas de contención tenían un efecto en el movimiento del virus, era esperable que el brote en algún momento debía alcanzar un punto máximo o cúspide que reflejaría el control sobre el crecimiento de casos.

La preparación para enfrentar la pandemia alentó al Ministerio de Salud de Costa Rica a acercarse a la Universidad de Costa Rica con la conformación de un equipo de especialistas en diversas disciplinas para la atención de análisis del comportamiento de los casos por coronavirus y el impacto en el uso de camas hospitalarias. El equipo de investigación se conformó con especialistas en gestión de riesgos, estadística, ciencias del comportamiento y procesos de simulación.

El trabajo interdisciplinario del equipo de especialistas permitió valorar el comportamiento de la curva de contagio por la COVID-19 mediante la implementación de las medidas de contención establecidas en el país. En una primera etapa las medidas promovieron el autocuidado mediante el lavado de manos, el distanciamiento físico y la prevención de evitar aglomeraciones, mientras que la segunda etapa se concentró en la reducción de la movilidad en el país mediante la instauración del trabajo remoto de centros escolares, la restricción vehicular, la disminución de la capacidad de espacios públicos y regulación de horarios en las playas, entre otras.

Durante los primeros tres meses de la epidemia el equipo de especialistas realizó al menos cinco informes técnicos que aportaron insumos para la toma de decisiones en el manejo de la epidemia. Los informes incluyeron información de las proyecciones de casos y demanda de camas, así como el comportamiento y análisis del número de reproducibilidad.

Las principales recomendaciones plasmadas en los informes evidenciaron que las intervenciones realizadas por el Ministerio de Salud de Costa Rica modificaron el comportamiento exponencial del contagio a una forma logística, lo cual se reflejó en la capacidad de dar atención a los pacientes con la COVID-19 en las redes hospitalarias. También el equipo de especialistas calculó el número de reproducibilidad en etapas iniciales de la pandemia 
(Nishiura 2013) demostrando que el valor funcionaba como evidencia de la descripción de la rapidez de la transmisión del virus entre personas.

En la emergencia pandémica es relevante la toma de decisiones basada en evidencia científica y técnica, lo cual significa que la captura de elementos sustentantes de las acciones transforma en retos los estudios y análisis realizados en tiempo real de la emergencia, por lo que es de vital importancia la incorporación, adaptación y calibración de modelos con resultados interpretables y vinculados a la mitigación de contagio (Roosa et al. 2020).

El presente estudio tiene la intención de informar sobre el comportamiento del estado inicial del contagio con los casos confirmados diariamente en el contexto de dos escenarios con y sin medidas de mitigación del contagio. También se describe cómo la información derivada de los análisis permitió la sustentación de intervenciones en el avance de la epidemia en cuanto al uso de camas hospitalarias.

\section{Modelos}

\section{Modelo de crecimiento exponencial}

El modelo exponencial (Paine et al. 2012) corresponde al conformado por dos parámetros, los cuales permiten establecer el comportamiento de los datos de los casos acumulados. El modelo captura el comportamiento en dos fases: en una primera fase los datos diarios crecen en función de un valor inicial y en una segunda etapa se acelera el crecimiento de casos. La expresión del modelo exponencial se describe como

$$
\mathrm{y}_{\mathrm{t}} \sim a e^{b t}
$$

donde $t$ representa el consecutivo de días y $y$ es la variable dependiente que representa la cantidad de casos acumulados y los parámetros $a$ y $b$ describen el comportamiento inicial y la rapidez del crecimiento, respectivamente. Otra versión del modelo exponencial que incluye un parámetro adicional se expresa como

$$
\mathrm{y}_{\mathrm{t}} \sim a e^{b t}+\mathrm{c}
$$

donde $c$ corresponde a la intersección con el eje de las ordenadas. 


\section{Modelo de crecimiento logístico}

El modelo logístico (Paine et al. 2012) se conforma de tres parámetros que permiten establecer el comportamiento de los datos en tres fases: en una primera fase los datos diarios crecen de forma lenta, en una segunda etapa se acelera el crecimiento de casos para luego entrar en la tercera fase donde se alcanza la capacidad de carga del modelo con un comportamiento asintótico. La expresión del modelo logístico se describe como

$$
\mathrm{y}_{\mathrm{t}} \sim \frac{\phi_{1}}{1+\mathrm{e}^{\frac{-\left(\mathrm{t}-\phi_{2}\right)}{\phi_{3}}}}
$$

donde los parámetros $\phi_{1}, \phi_{2}$ y $\phi_{3}$ describen el comportamiento de capacidad de carga, simetría del comportamiento y la rapidez del crecimiento, respectivamente.

\section{Modelo de Richards}

El modelo de Richards (Tjørve y Tjørve 2010) permite observar el comportamiento subexponencial y la potencial transición desde el comportamiento logístico al exponencial. El modelo de Richards se expresa como

$$
\mathrm{y}_{\mathrm{t}} \sim \frac{\mathrm{A}}{\left(1+\mathrm{m} \cdot e^{-k(t-i)}\right)^{\frac{1}{m}}}
$$

donde los cuatro parámetros describen la asíntota superior $A$, el mínimo o asíntota inferior $k$, el factor de crecimiento $m$, y el máximo relativo del factor de crecimiento $i$. El modelo de Richards subsume otros modelos como el modelo de crecimiento logístico y el de Gompertz. Si $m=1$, se obtiene el modelo logístico y cuando $\mathrm{m}=\frac{1}{10}$ se deriva el modelo de Gompertz (Oswald et al. 2012). 


\section{Método}

\section{Supuestos}

Los análisis que se realicen con los modelos previamente mencionados asumen que el comportamiento del crecimiento de casos se da a partir de los siguientes supuestos:

1. Hay al menos una persona contagiada en la población.

2. Las personas contagiadas y no contagiadas tienen contacto entre ellas.

3. Hay una cantidad de personas sin contagio que potencialmente se contagiarán.

4. Las medidas sanitarias permiten el control del contagio por lo que se tendrá un máximo de contagios menor que la población susceptible.

5. Las personas que hayan estado en contacto con las personas contagiadas deben identificarse rápidamente.

6. El crecimiento exponencial y las medidas sanitarias compiten entre sí.

\section{Datos}

Los datos corresponden a los casos confirmados desde el 6 de marzo al 6 de junio de 2020 por contagio de la COVID-19 en Costa Rica. Los datos han sido registrados a partir de los reportes diarios emitidos por el Ministerio de Salud, tanto vía conferencia de prensa en formatos de imagen y más recientemente en formatos de hojas de cálculo que facilitan la transferencia de la información.

\section{Estimación de modelos}

Se realizó la estimación de cuatro modelos con el método de mínimos cuadrados no lineales: logístico, exponencial, Richards y Gompertz. Los modelos se estiman con los algoritmos de mínimos cuadrados (Florent et al. 2015 ) implementados en la plataforma de $R$ en la paquetería FlexParamCurve (Oswald et al. 2012). Dado que los modelos requieren valores iniciales para la estimación, se utilizó las funciones self-starting, las cuales permiten 
el proceso de arranque en la estimación de los parámetros. A partir de los parámetros estimados, se generaron los pronósticos, los intervalos de confianza y los de predicción de las estimaciones, los cuales se calculan a partir de la expansión de Taylor de primer y segundo orden y simulación Montecarlo implementados en la librería propagate (Spiess 2018).

\section{Simulación de demanda de camas hospitalarias}

Los valores de casos acumulados proyectados se incorporaron a un modelo de simulación aleatoria de eventos discretos que generó la demanda de camas hospitalarias. En el modelo los incrementos y los decesos de casos no generaron el alcance de un estado estacionario tal como ocurre en el modelado de procesos de servicios o de producción. El código de generación de las camas requeridas según la proyección se implementó en lenguaje Python.

A partir de estudios preliminares en Italia de Grasselli, Pesenti y Ceconi (2020) y Livingston y Bucher (2020) se consideró que en la proyección de uso de camas el $80 \%$ de las personas contagiadas por la COVID-19 no requerían hospitalización, el $15 \%$ de los infectados pasaban a una cama de atención general y el $5 \%$ requería una cama o unidad de cuidados intensivos (UCI). Además, se estableció una estadía promedio de 6 días (desviación estándar de 3,6) para las personas que requieren atención general y un tiempo promedio de 11 días (desviación estándar de 6,5) para la estancia en unidad de cuidados intensivos (UCI).

El algoritmo inicia generando una cantidad de pacientes según una distribución normal estándar e incorpora la cantidad de casos proyectados diariamente. Luego, a cada paciente se le genera un día de hospitalización y se le asigna una de las tres rutas según la probabilidad de la necesidad de atención en cama general, cuidados intensivos o por no requerir hospitalización. Finalmente, se obtiene el número de camas requeridas en función de la cantidad contagios pronosticados. El proceso es iterativo y se repite 1000 veces para calcular el promedio y la desviación estándar de la cantidad de camas requeridas. La cantidad alta de réplicas compensaron la limitada cantidad de días de pronóstico disponible. 


\section{Estimación del número de reproducibilidad}

La estimación de los pronósticos se acompañó de un análisis de la tasa de reproducibilidad, $\mathrm{R}_{t}$ con dependencia en el tiempo (Cori et al. 2013). El número de reproducibilidad indica la cantidad de personas que se contagian a partir de una persona infectada (Nishiura 2013). La tasa permite observar, a nivel general, si las medidas de contención han producido algún cambio en el comportamiento de la transmisión del virus puesto que el $\mathrm{R}_{\mathrm{t}}$ se calcula posterior a que se hayan adoptado medidas de contención.

Los valores del $\mathrm{R}_{\mathrm{t}}$ superiores a 1 indican que el comportamiento es acelerado y los valores por debajo de 1 representan que el contagio empieza a desacelerarse. El $\mathrm{R}_{\mathrm{t}}$ se calculó con estimación bayesiana con rezagos de 7 días que siguen una distribución gamma. El análisis se implementó en el programa R con la librería EpiEstim (Cori et al. 2020).

\section{Conformación de un equipo de especialistas}

El Ministerio de Salud de Costa Rica solicitó a la Universidad de Costa Rica la conformación de un equipo de especialistas en diversas disciplinas para la atención de análisis del comportamiento de los casos por coronavirus y el impacto en el uso de camas hospitalarias. El equipo de investigadores se conformó con especialistas en gestión de riesgos, estadística, ciencias del comportamiento y procesos de simulación.

El equipo realizó al menos 5 informes técnicos que aportaron insumos para la toma de decisiones en el manejo de la epidemia. Los tres primeros informes, con información de las proyecciones y demanda de camas, se realizaron el 8, 14 y 23 de abril de 2020, mientras que el cuarto reporte del 10 de mayo de 2020 presentó el comportamiento y análisis del número de reproducibilidad. Finalmente, el quinto informe se dio el 5 de junio de 2020 con un análisis por semanas del índice de transmisibilidad. 


\section{Resultados}

Los resultados se muestran en tres vertientes. En la primera se presentan los parámetros estimados a partir de los modelos y resultados de predicción a partir de los distintos modelos. Posteriormente, se muestran los pronósticos de las demandas de camas hospitalarias. Seguidamente, se describe el comportamiento del número de reproducibilidad. Finalmente, se muestra la optimización de los modelos y simulaciones para la toma de decisiones.

La figura 1 muestra el análisis con los datos de los primeros 17 días con el modelo exponencial, el cual evidencia la posible tendencia que tomaría el comportamiento del coronavirus en Costa Rica. Según se aprecia en la figura 1, en los primeros 10 días se marcó una tendencia exponencial: sin embargo, con la declaratoria del estado de emergencia nacional y de confinamiento a partir del día 11, la tendencia de casos reales se alejó de la forma exponencial, lo cual sugirió un potencial efecto de las medidas sanitarias y por ende la presencia de un comportamiento logístico. Este hecho se observa en la curva de casos diarios observados, la cual presenta un crecimiento hasta el día 22 o 23 y luego decrece hasta el día 50 .

Los resultados desplegados en la figura 1 marcaron el camino para realizar el modelado subsecuente con los cuatro modelos. La tabla 1 muestra los parámetros estimados a partir de los modelos. Los resultados se presentan en escenarios que permiten valorar comportamiento de los datos. El escenario pesimista está estipulado por un crecimiento acelerado bajo el modelo exponencial y el escenario optimista está representado por el modelo logístico donde se asume que hay un control sobre el ritmo de aparición de casos. En el medio de los escenarios optimista y pesimista se encuentran los modelos que marcan una potencial transición entre los escenarios. Los escenarios intermedios están representados con los modelos de Richards y Gompertz.

Los parámetros estimados en la tabla 1 permiten observar que los valores obtenidos con la serie de datos de 1-34 plantearon un punto de inflexión entre el día 22 y 23. Este punto de inflexión denota un primer pico de casos que marca la tendencia de la primera ola del brote. El valor de la asíntota horizontal para los datos 1-34 evidenció que la capacidad máxima de casos acumulados estaría alrededor de los 668 con el modelo de Gompertz. Los casos observados acumulados rondaron los 700 al momento en que la primera ola terminó su caída entre los días 49 a 56. 
Guaner Rojas, Rodolfo Romero, Ronny Pacheco, Carlos Villalobos y Agustín Gómez

Figura 1

Panel superior: estimación del modelo exponencial para los casos acumulados en los primeros 17 días de epidemia. Panel inferior: curva de casos diarios observados
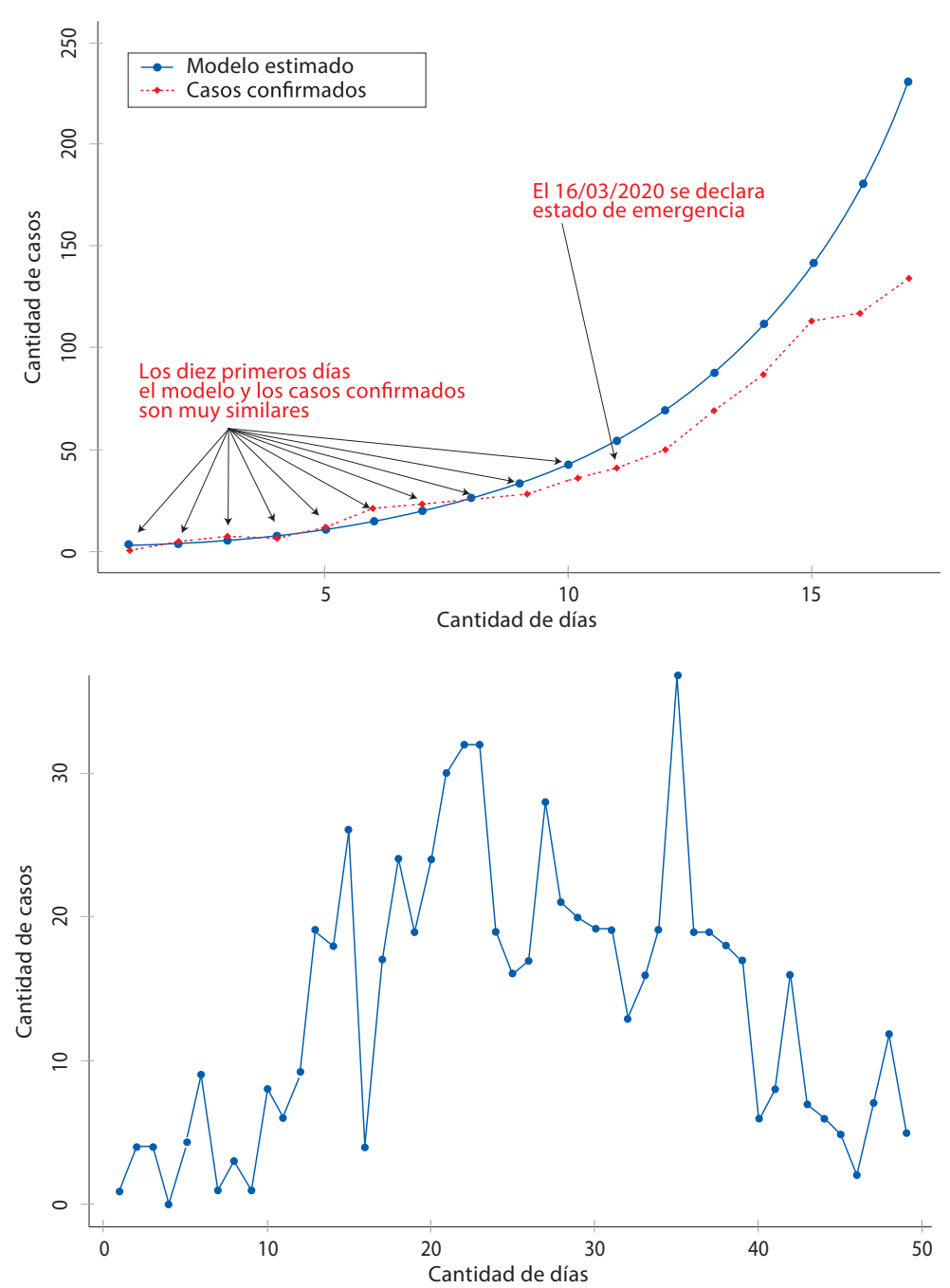

Elaboración propia. 
Los valores del parámetro de crecimiento en el modelo exponencial tendieron a disminuir desde 0,08 a 0,05 conforme se fueron utilizando más datos en los modelos. Este comportamiento del modelo exponencial se corresponde con la desaceleración de la aparición de casos después del día 23 del inicio de la pandemia.

Tabla 1

\section{Parámetros de los estimados en tres distintos momentos}

\begin{tabular}{|c|c|c|c|c|c|c|c|c|}
\hline \multirow{2}{*}{ Días } & \multirow{3}{*}{ Modelo } & \multicolumn{4}{|c|}{ Parámetros } & \multirow{2}{*}{ Modelo } & \multicolumn{2}{|c|}{ Parámetros } \\
\cline { 3 - 6 } \cline { 5 - 8 } & & $\mathbf{A}$ & $\mathbf{k}$ & $\mathbf{i}$ & $\mathbf{m}$ & & $\mathbf{a}$ & $\mathbf{b}$ \\
\hline \multirow{4}{*}{$1-34$} & Logístico & 539,97 & 0,10 & 22,02 & 1 & Exponencial & 35,56 & 0,08 \\
& Richards & 596,38 & 0,14 & 22,17 & 0,45 & & & \\
& Gompertz & 668,85 & 0,20 & 22,56 & 0,1 & & & \\
\hline \multirow{4}{*}{$1-40$} & Logístico & 653,15 & 0,16 & 25,23 & 1 & Exponencial & 52,41 & 0,07 \\
& Richards & 838,68 & 0,07 & 23,91 & $-0,07$ & & & \\
& Gompertz & 782,91 & 0,09 & 24,10 & 0,1 & & & \\
\hline \multirow{4}{*}{$1-49$} & Logístico & 695,00 & 0,15 & 26,27 & 1 & Exponencial & 87,46 & 0,05 \\
& Richards & 761,35 & 0,10 & 23,72 & 0,11 & & & \\
& Gompertz & 762,15 & 0,09 & 23,70 & 0,1 & & & \\
\hline
\end{tabular}

Elaboración propia.

La figura 2 muestra la transición potencial desde un escenario con comportamiento logístico (línea azul) hasta un escenario con comportamiento exponencial (línea negra) y dos escenarios intermedios a partir de la serie de datos observados desde el día 1 al día 34 . El crecimiento de casos diarios sigue un comportamiento con forma sigmoide desde el día 1 hasta el 49, ubicándose ya sea sobre la curva logística o la de Gompertz. En el panel inferior de la figura 3 las curvas de Gompertz y Richards coinciden en el pronóstico de los casos.

Al día 34, el crecimiento de los datos se ubica sobre el escenario intermedio representado por la curva de Gompertz, mientras que al día 40 los casos acumulados se mantuvieron sobre la curva de Richards y al día 49 retomó la forma de Gompertz. El mantenimiento de la forma sigmoide es un indicativo 
de que las medidas adoptadas por el Ministerio de Salud mantuvieron cierto control en el crecimiento de los casos.

Figura 2

Proyección de casos acumulados según cuatro modelos: exponencial, Gompertz, Richards y logístico. Panel izquierdo: datos 1-34 días. Panel derecho: datos 1-49 días

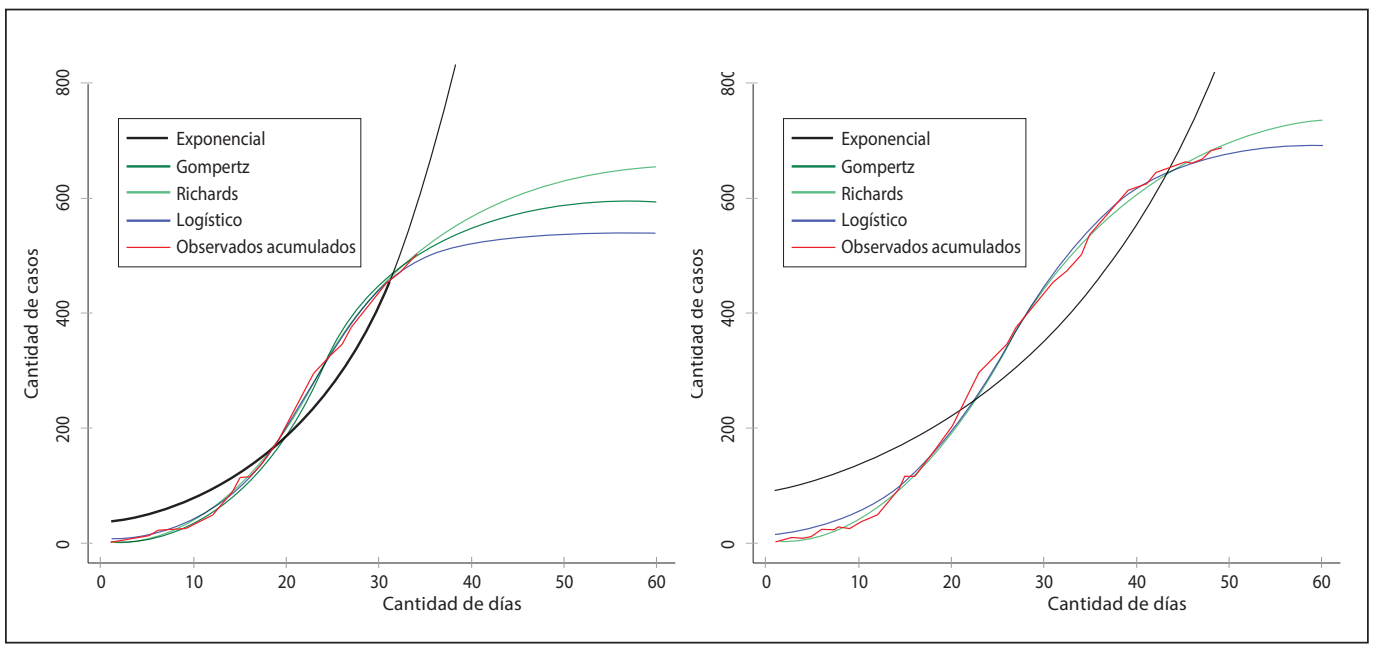

Elaboración propia.

La tabla 2 muestra los valores de la demanda de camas pronosticadas según los escenarios de análisis y tipos de camas. La ocupación real de las camas de cuidados intensivos no superó los 14 días en el período de los primeros tres meses de la pandemia. La cantidad de camas de cuidados intensivos proyectadas tendió a disminuir conforme se avanzó en el tiempo puesto que conforme se emplearon más datos de la serie de casos reportados se requirió una mayor cantidad de días para alcanzar la demanda máxima de camas.

En concordancia con el comportamiento de los casos con las curvas logística o Gompertz, se observa que las camas UCI proyectadas con la serie de días 1-34 permitió pronosticar el comportamiento real de las camas UCI. El modelo logístico estableció la necesidad máxima de 17 camas en 10 días, mientras que la estimación en el escenario Gompertz fue de 23 camas en 19 días. 
Tabla 2

\section{Pronóstico de camas requeridas según tipo de cama}

\begin{tabular}{|c|c|c|c|c|}
\hline Días & Escenario & Tipo de cama & $\begin{array}{c}\text { Cantidad de camas } \\
\text { requeridas }\end{array}$ & $\begin{array}{l}\text { Días para demanda } \\
\text { máxima }\end{array}$ \\
\hline \multirow{3}{*}{$1-34$} & Logístico & $\begin{array}{l}\text { General } \\
\text { UCl }\end{array}$ & $\begin{array}{l}17 \\
23\end{array}$ & $\begin{array}{l}10 \\
19\end{array}$ \\
\hline & Gompertz & $\begin{array}{l}\text { General } \\
\text { UCI }\end{array}$ & $\begin{array}{l}18 \\
23\end{array}$ & $\begin{array}{l}10 \\
20\end{array}$ \\
\hline & Exponencial & $\begin{array}{l}\text { General } \\
\text { UCl }\end{array}$ & $\begin{array}{l}212 \\
260\end{array}$ & $\begin{array}{l}36 \\
36\end{array}$ \\
\hline \multirow{3}{*}{$1-40$} & Logístico & $\begin{array}{l}\text { General } \\
\text { UCI }\end{array}$ & $\begin{array}{l}21 \\
27\end{array}$ & $\begin{array}{l}11 \\
22\end{array}$ \\
\hline & Gompertz & $\begin{array}{l}\text { General } \\
\text { UCl }\end{array}$ & $\begin{array}{l}32 \\
43\end{array}$ & $\begin{array}{l}31 \\
31\end{array}$ \\
\hline & Exponencial & $\begin{array}{l}\text { General } \\
\text { UCl }\end{array}$ & $\begin{array}{r}92 \\
114\end{array}$ & $\begin{array}{l}30 \\
30\end{array}$ \\
\hline \multirow{3}{*}{$1-49$} & Logístico & $\begin{array}{l}\text { General } \\
\text { UCl }\end{array}$ & $\begin{array}{l}20 \\
26\end{array}$ & $\begin{array}{l}12 \\
22\end{array}$ \\
\hline & Gompertz & $\begin{array}{l}\text { General } \\
\text { UCl }\end{array}$ & $\begin{array}{l}26 \\
35\end{array}$ & $\begin{array}{l}20 \\
29\end{array}$ \\
\hline & Exponencial & $\begin{array}{l}\text { General } \\
\text { UCl }\end{array}$ & $\begin{array}{l}53 \\
70\end{array}$ & $\begin{array}{l}30 \\
30\end{array}$ \\
\hline
\end{tabular}

Elaboración propia.

La figura 3 muestra la estimación del número de reproducibilidad según hitos relevantes en el período del 1-40. El comportamiento del índice durante los primeros tres meses evidenció que las medidas adoptadas en los primeros 20 días de la epidemia tuvieron control sobre el crecimiento de casos. Al inicio de la epidemia el $\mathrm{R}_{\mathrm{t}}$ tomó valores bastante altos alrededor de 3,5 reflejando la rapidez con la que se movía el virus entre el caso primario y los casos secundarios, sin embargo, con el paso de los días el valor tendió a estabilizarse y descendió a valores cercanos a 1. 


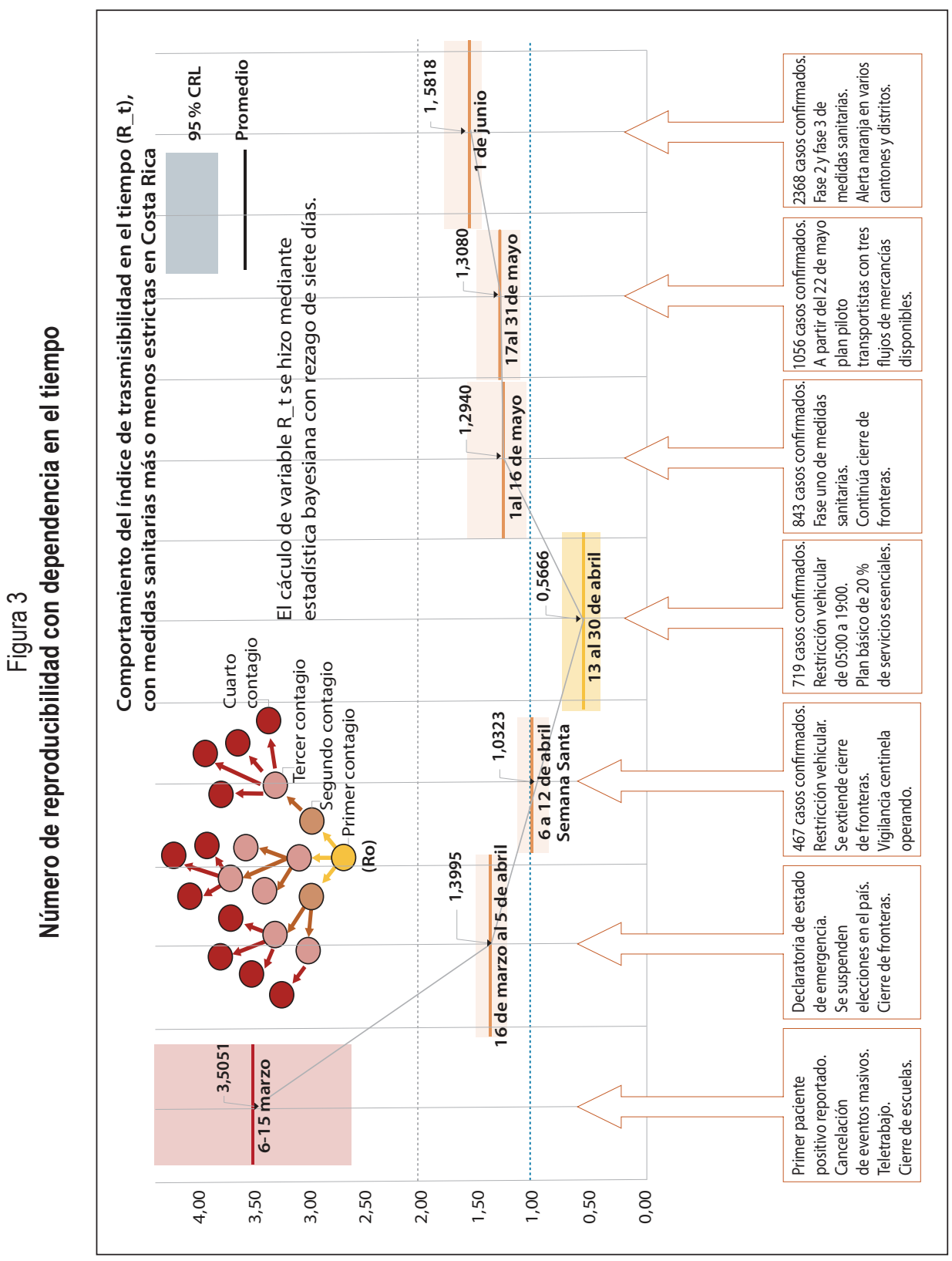

$\frac{\pi}{0}$
$\frac{0}{2}$
$\frac{0}{1}$
$: \frac{0}{0}$
$\frac{\pi}{0}$
$\frac{0}{\pi}$
$\frac{\pi}{4}$ 
El equipo de investigación generó como complemento a los modelos de aproximación y simulación en la primera etapa del análisis de los datos antes descritos, la optimización por medio de informes técnicos la forma de presentar gráficos y los análisis para interpretación menos compleja y de mayor facilidad de acceso para los tomadores de decisiones. Esto con el objetivo de aplicar métodos de decisión optimizados, lo cual permite contar con un proceso sistemático, consistente y conciso, que faculte una toma decisiones lo más efectiva posible.

La figura 4 muestra un ejemplo de la optimización de la información mediante gráficos. El gráfico de la figura 4 presenta un panel de termómetro que representa los límites de los distintos niveles de alerta según la cantidad de casos diarios, semanales y ocupación de camas. Por ejemplo, durante los primeros tres meses una cantidad de casos superior a 33 y una ocupación de camas mayor a 70 marcó la caracterización en un nivel 3 de alerta.

Figura 4

Ejemplo de gráfico optimizado de resultados de simulación de camas UCl para toma de decisiones de autoridades sanitarias

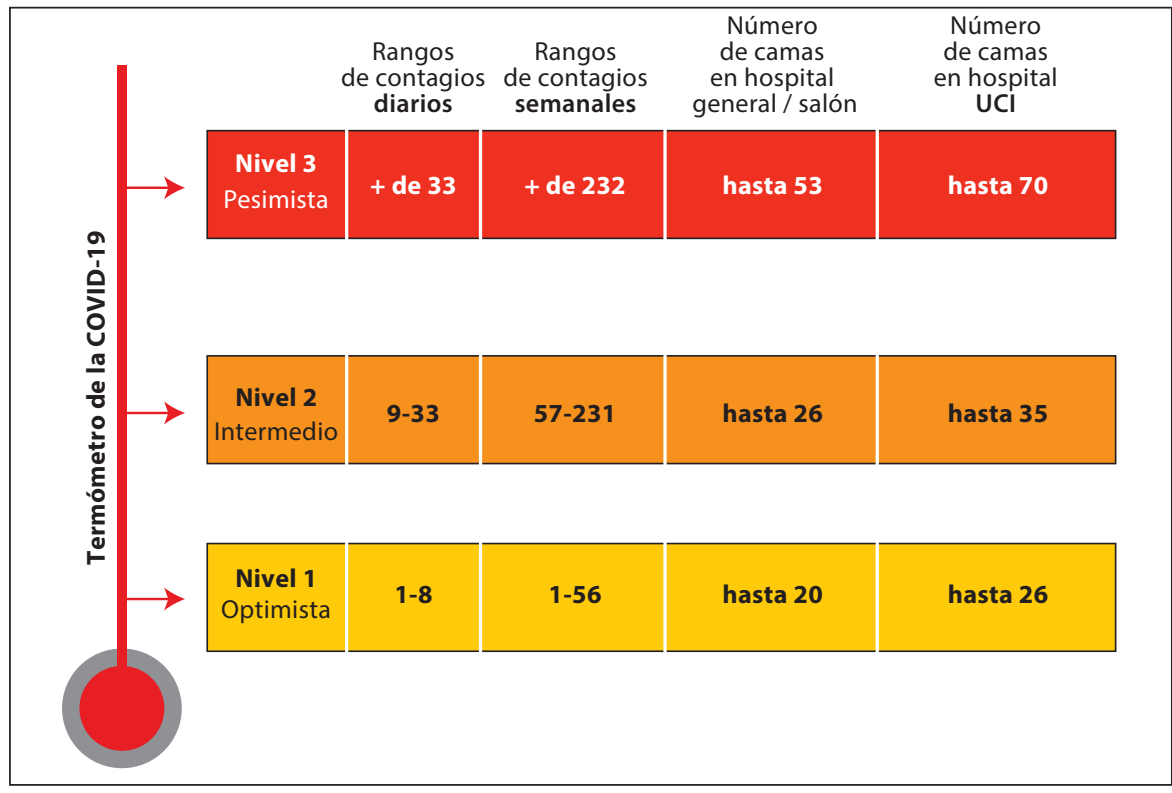

Elaboración propia. 


\section{Conclusiones}

El presente estudio ha mostrado uno de los procedimientos de atención ante la emergencia de la COVID-19 en Costa Rica. A partir de la articulación y conformación de un equipo interdisciplinario de investigación se generaron estudios alrededor del comportamiento del virus y el impacto en el uso de camas hospitalarias de uso general y de cuidados intensivos. El comportamiento de la curva de contagio se estudió mediante el modelado estadístico con modelos exponenciales y logísticos, y con la tasa de reproducibilidad con dependencia en el tiempo.

El estudio de la forma de la curva de contagio permitió generar los pronósticos de casos y la alimentación de un modelo de simulación aleatoria con un diseño de tres rutas para la proyección de la demanda potencial de camas hospitalarias y períodos aproximados de 30 días hacia adelante del último día de casos confirmados por la COVID-19.

Los análisis desarrollados con los datos 1-49 permiten observar que los casos confirmados siguieron un comportamiento logístico, tal como se esperaba según el análisis con los primeros 17 días de la serie y los pronósticos realizados con los datos 1-34. El comportamiento puede tomarse como un indicativo de que las medidas sanitarias surtieron efecto en evitar que los casos crecieran rápidamente. El comportamiento logístico fue confirmado por el cálculo de la tasa de transmisibilidad, la cual se redujo notoriamente cuando se aplicaron medidas más estrictas de contención.

Las interpretaciones de los análisis realizados permiten anticipar determinadas acciones a nivel nacional para el comportamiento de la segunda ola. Este estudio contempló un análisis de la primera etapa de la pandemia por lo que en un futuro estudio será posible trasladar la metodología al análisis de la segunda ola de contagio. Una subsecuente línea de trabajo incorporará la generación de un estudio que manifieste el comportamiento de la curva de contagio en segmentos espaciales o unidades geográficas que estén previamente definidas.

La variedad de información derivada de los análisis ayuda a los tomadores de decisiones en la medida en que los resultados se presenten de manera que pueda ser accedida de forma ágil y fácil. Por ejemplo, el uso de plataformas tecnológicas permite reunir en un solo paquete todos los resultados 
relevantes, los cuales permitirían a los tomadores de decisiones tener en primera línea de forma sencilla y rápida el acceso a los indicadores y reportes técnicos.

En el marco de la pandemia por la COVID-19, dado el alto nivel de incertidumbre y variabilidad del fenómeno poco conocido, el tomador de decisiones requiere una estructura de datos y una metodología que permita interpretar fácilmente lo que está pasando y lo que puede pasar para ir articulando e incorporando más variables a su análisis de manera paulatina. Dicho de otra manera, las personas encargadas de la toma de decisiones son racionales e intentan ser cada vez más proactivas en esta tarea.

Finalmente, este artículo como complemento a la generación de los modelos de aproximación y simulación en la primera etapa del proyecto antes descritos, ha permitido evidenciar cómo un equipo de profesionales ha optimizado por medio de informes la forma de presentar gráficos y los análisis para interpretación menos compleja. Esto con el objetivo de aplicar métodos de decisión potenciados, lo cual permite contar con un proceso sistemático, consistente y conciso, que faculte una toma decisiones lo más efectiva posible.

\section{Referencias}

Baty, Florent, Christian Ritz, Sandrine Charles, Martin Brutsche, Jean-Pierre Flandrois y Marie-Laure Delignette-Muller. 2015. "A Toolbox for Nonlinear Regression in R: The Package nlstools". Journal of Statistical Software 66 (5): 1-21. http://www.jstatsoft.org/.

Chaves, Luis Fernando, Lisbeth Hurtado, Melissa Ramírez Rojas, Mariel Friberg, Rodrigo Marín Rodríguez y María Ávila-Agüero. 2020. "Covid-19 Basic Reproduction Number and Assessment of Initial Suppression Policies in Costa Rica". Mathematical Modelling of Natural Phenomena 15 (32): 1-13. https://doi.org/10.1051/mmnp/ 2020019.

Chowell, Gerardo, Lisa Sattienspiel, Sweta Bansai y Cécile Viboud. 2016. "Mathematical Models to Characterize Early Epidemic Growth. A Review". Physics of Life Reviews 18 (1): 66-97. 10.1016/j.plrev.2016.07.005.

Cori, Anne, Simon Cauhemez, Neil Fergunson, Christophe Freiser, Elizabeth Dahlqwist, Alex Demarsh, Thibaut Jombart, Zhian Kamvar, Justin Lessler, Shikun Li, Jonathan Polonsky, Jake Stockwin, Robin Tompson y Robina van Galeen. 2020. Estimate Time Varying Reproduction Numbers from Epidemic Curves. R Project for Statistical Computing. R package version 2.2.4. PC y Mac OS. 
Cori, Anne, Neil Ferguson, Christophe Fraser y Simon Cauchemez. 2013. "A New Framework and Software to Estimate Time-Varying Reproduction Numbers During Epidemics". Practice of Epidemiology 178 (9): 1505-1512. 10.1093/aje/kwt133.

Grasselli, Giacomo, Antonio Pesenti y Maurizio Ceconi. 2020. "Critical Care Utilization for the COVID Outbreak in Lombardy, Italy". American Medical Association 323 (16): 15451546. 10.1001/jama.2020.4031.

Livingston, Edward, y Karen Bucher. 2020. "Coronavirus Disease 2019 (COVID-19) in Italy". American Medical Association 323 (14): 1335-1335. 10.1001/jama.2020. 4344.

Ministerio de Salud de Costa Rica. 2021. "Comunicados de prensa COVID-19". Accedido enero. https://bit.ly/3rMKj13.

Nishiura, Hiroshi. 2010. "Correcting the Actual Reproduction Number: A Simple Method to Estimate R0 from Early Epidemic Growth Data". International Journal of Environmental Research and Public Health 7: 291-302. 10.3390/ijerph7010291.

Oswald, Stephen, Ian Nisbet, Andre Chiaradia y Jennifer Arnold. 2012. "Flexparamcurve: R Package for Flexible Fitting of Nonlinear Parametric Curves". Methods in Ecology and Evolution 3: 1073-1077. 10.1111/j.2041-210X.2012. 00231.x.

Paine, Timothy, Toby Marthews, Deborah Vogt, Drew Purves, Mark Rees, Andy Hector y Lindsay Turnbull. 2012. "How to Fit Nonlinear Plant Growth Models and Calculate Growth Rates: An Update for Ecologists". Methods in Ecology and Evolution 3: 245-256.

Roosa, Kimberlyn, Yiseul Lee, Ruiyan Luo, Alexander Kirpich, Richard Rothenberg, James Hyman, Ping Yan y Gerardo Chowell. 2020. "Short-term Forecasts of the COVID-19 Epidemic in Guangdong and Zh ejiang, China: February 13-23, 2020". Journal of Clinical Medicine 9 (596): 1-9. 0.3390/jcm9020596.

Spiess, Andrej-NiKolai. 2018. Propagation of Uncertainty Using Higher-Order Taylor Expansion and Monte Carlo Simulation. The R Project for Statistical Computing. R package version 1.0.6. PC y Mac. Montecarlo. Accedido enero de 2021. rdrr.io/cran/propagate/ $\mathrm{man} /$ propagate.html.

Tjørve, Even, y Kathleen Tjørve. 2010. "A Unified Approach to the Richards-model Family for use in Growth Analyses: Why We Need Only Two Model Forms". Journal of Theoretical Biology 267: 417-425.

Torabipour, Amin, Hojjat Zeraati, Mohammad Arab, Arash Rashidian, Sari Akbari, Ali Sarzaiem y Mahmuod Reza. 2016. "Bed Capacity Planning Using Stochastic Simulation Approach in Cardiac-surgery Department of Teaching Hospitals, Tehran, Iran”. Iranian Journal of Public Health 45 (9): 1208-1216. http://ijph.tums.ac.ir. 